WORLD VIEW

\title{
Quest for quality care and patient safety: the case of Singapore
}

\section{K Lim}

Qual Saf Health Care 2004;13:71-75. doi: 10.1136/qshc.2002.004994

Quality of care in Singapore has seen a paradigm shift from a traditional focus on structural approaches to a broader multidimensional concept which includes the monitoring of clinical indicators and medical errors. Strong political commitment and institutional capacities have been important factors for making the transition. What is still lacking, however, is a culture of rigorous programme evaluation, public involvement, and patient empowerment. Despite these imperfections, Singapore has made considerable strides and its experience may hold lessons for other small developing countries in the common quest for quality care and patient safety.

Correspondence to: M K Lim, Department of Community, Occupational and Family Medicine, Faculty of Medicine, National University of Singapore, MD3, 16 Medical Drive, Singapore 117597 ;

coflimmk@nus.edu.sg
Q uality care is by no means a new concept in Singapore, where it has long been assumed to be an implicit goal of the healthcare system. What is new is the adoption of a systematic and scientific approach to its measurement and management. ${ }^{1}$ Likewise, the recognition of "medical errors" as a systemic problem requiring systemic solutions ${ }^{2}$ is a recent and positive development.

The impetus for change, however, is not coming from public pressure for hospitals to be held accountable for the quality of care they deliver, but from a paternalistic government that strives to be proactive in most matters. The government-as the regulator, major purchaser, and major public provider-is pushing for change on behalf of Singapore's non-vociferous, law abiding citizens. Although it has not been consciously pursued as such, the evolutionary path taken by the largely "top down" quality healthcare movement can be described in Donabedian terms. ${ }^{3}$ Initially focused on structures, it has recently turned on processes and outcomes.

This paper documents Singapore's initiatives in quality health care and patient safety and draws some lessons which may be useful to others who share this common quest.

\section{INITIAL FOCUS ON SERVICE QUALITY}

First time visitors to Singapore, a tiny island of $660 \mathrm{sq} \mathrm{km}$, are likely to be impressed by its safety, cleanliness, and sheer efficiency. Once a British colonial outpost, the bustling city state now ranks among the richest countries in the world with a per capita GDP of US $\$ 26500^{4}$ (purchasing power parity) which exceeds that of the leading European countries. Its 4 million inhabitants enjoy a good quality of life. Average life expectancy is 78 years and infant mortality is 3.0 per 1000. Details of the Singapore healthcare system are shown in box $1 .^{5}$

Oiling the wheels of success and striving for "excellence" has become something of a national obsession for "Singapore Inc". The concepts and methods of the modern quality movement inspired by Deming, Juran, Crosby, Ishikawa, Taguchi and others ${ }^{67}$ are well known and in evidence everywhere-in business, commerce and government. The first National Quality Control Circle Convention was held in 1982. Since then, healthcare managers have conscientiously adapted total quality management and continuous quality improvement principles and applied them in the health services sector. Today, no hospital or specialist medical centre-either in the public or private sector-is without a quality committee of sorts. Variously called the "Quality Forum", "Quality Council", or simply "Quality Committee", these committees, usually chaired by the hospital's Chief Executive Officer, oversee all organization wide, quality related initiatives and keep track of key performance indicators. Quality Circles and Work Improvement Teams regularly brainstorm workflow problems or service quality related matters, their efforts bearing fruit in many ways such as reduced admission processing times, reduced patient waiting times at specialist outpatient clinics, and more efficient management of bed occupancy.

Employees routinely undergo service improvement courses (for example, "Six Sigma", "Seven Habits", "Team Building", "Statistical Process Control", "Internal Quality Audit") and outstanding performers are highlighted in newsletters and recognized during annual Quality Conventions. Many hospitals have been ISO 9K, $14 \mathrm{~K}$ or $18 \mathrm{~K}$ certified, and a few have gone on to achieve the Singapore Quality Class (the local version of the Baldrige Award or the European Quality Award) launched in 1994.

Service quality, however, is not to be confused with clinical quality. The latter has largely been assumed-partly because of the high public esteem enjoyed by the medical profession, but mostly because healthcare managers lacked the knowledge and tools for its objective measurement and evaluation. All this, however, is fast changing.

\section{PARADIGM SHIFT: FOCUS ON CLINICAL QUALITY}

With growing awareness of the advances in the science of performance measurement and in 
Box 1 Singapore's healthcare system

- Singapore inherited a British style, largely tax based, and publicly provided healthcare system at independence in 1965. Over the years it has evolved under a pragmatic government bent on eschewing egalitarian welfarism in favour of market mechanisms to allocate scarce healthcare resources.

- Today, health care is financed by a combination of state subsidies $(25 \%)$, employer benefits $(35 \%)$, out of pocket payments $(25 \%)$, compulsory medical savings for acute care expenses (8\%), risk sharing for catastrophic illnesses $(2 \%)$, and private health insurance $(5 \%)$ (Ministry of Health, unpublished data, 2000). National health care expenditure has remained fairly constant at $3 \%$ of GDP over the last two decades. ${ }^{23}$ The WHO 2000 Report ranked Singapore sixth (out of 191) in "overall health system performance" ${ }^{\prime \prime}{ }^{24}$

- Patients have complete freedom of choice of providers. Primary health care is easily accessible through private medical practitioners (80\%) and government outpatient polyclinics $(20 \%)$. There are 26 well equipped hospitals and specialty centres providing 11798 beds (ratio of 3.7 beds per 1000 population). Eight public hospitals and five specialty centres (ranging from 80 to 3110 beds) account for $80 \%$ of the beds while 13 private hospitals (from 25 to 500 beds) account for the remainder. Three private hospital chains are listed on the Stock Exchange of Singapore.

- Since 1985 every public sector hospital has been "restructured" - the latter term referring to the granting of autonomy in operational matters so as to inject private sector efficiency and financial discipline, but with the government retaining $100 \%$ ownership of the hospitals. Initially managed by a monolithic government company, the restructured hospitals underwent further reorganization in 2000, splitting into two competing clusters - the National Healthcare Group and the Singapore Health Services-but ultimately reporting to the $\mathrm{MOH}$.

- Singapore's doctors enjoy a high reputation, as attested by the steady streams of well heeled patients who fly in from the surrounding region for medical care. In 2000 an estimated 150000 foreign patients sought treatment in Singapore. ${ }^{25}$ Recently, a governmental Economic Review Committee has set a target of one million foreign patients a year in 10 years' time, which would bring in an estimated $\$ 3$ billion annually and create 13000 jobs. ${ }^{26}$ As Singapore strives to become a regional medical hub of excellence, a major challenge will be to ensure uncompromising standards in the quality and safety of health care that is both affordable and accessible to all Singaporeans.

tandem with trends in the western industrialized countries, ${ }^{8-10}$ healthcare providers are beginning to adopt a more systematic and scientific approach to clinical quality. Hospitals in both the public and private sectors now employ teams of full time quality managers to measure clinical processes and outcomes instead of simply leaving it to individual doctors to decide what works best for their patients. The Ministry of Health $(\mathrm{MOH})$, together with its two healthcare clusters and the Singapore Medical Association, even played host to the
Box 2 Singapore's regulatory framework for quality: Relevant Health Regulations

Quality assurance committees

- Mandatory for all hospitals (1980)

National medical audit programme

- Clinical Audit (1999)

- Maryland Quality Indicator Project (2000)

- Specialty Specific Clinical Indicators (2003)

- Tissue Audit (?)

- Mortality (?)

- Medical Errors/Near Misses, Complaints (2002)

- Disease Registers (2003)

2nd Asia Pacific Forum on Quality Improvement in Health Care in September 2002, organised with the help of the BMJ Publishing Group and the Institute for Healthcare Improvement.

The Minister of Health has promised in Parliament that "by the end of 2003 there will be hospital wide and specialty specific clinical indicators to monitor and improve clinical service provision". ${ }^{11}$ Reflecting the demand for acquiring the knowledge and skills needed for clinical performance evaluation, an annual 5 day course conducted by the National University of Singapore over the past 4 years on "Measuring and Managing Quality of Care" has invariably been oversubscribed.

It is worth noting, however, that until about 1999 the $\mathrm{MOH}$ led efforts focused almost exclusively on structural approaches-the assumption being that good doctors and facilities would be conducive to good processes and outcomes. This assumption may not hold true, of course, but the strong "structural" emphasis on regulation (boxes 2 and 3), organization, and management (box 1) has worked well for Singapore. Indeed, it has provided a strong foundation on which to build the more recent initiatives (box 4).

\section{STRONG REGULATORY ENVIRONMENT}

Singapore's only western style medical school, established in 1905, produces approximately 230 doctors a year. Standards are high, as evidenced by the longstanding recognition of its medical degree by the United Kingdom's General Medical Council and Royal Colleges of Physicians and Surgeons. Healthcare professionals are required by law to register with the relevant professional bodies-namely, Singapore Medical Council, Singapore Nursing Board, Singapore Dental Board, and Singapore Pharmacy Board. Each is charged with protecting the public and empowered with appropriate professional disciplinary mechanisms, including the power to de-register.

Forty percent of registered medical practitioners are specialists who are certified by a Specialist Accreditation Board appointed by the MOH. Through its Health Manpower Development Program established in 1980, the $\mathrm{MOH}$ sponsors public sector doctors and nurses for exposure and training at leading healthcare centres of the world, and brings renowned overseas experts to Singapore to share their expertise. A Joint Committee on Specialist Training (with representation from the Graduate School of Medical Studies and the Academy of Medicine of Singapore) oversees 35 Specialist Training Committees which, in turn, oversee specialist medical training in the respective fields.

Voluntary Continuing Medical Education (CME) has been ongoing for many years but participation has been patchy. In 1998 only $10 \%$ of specialists qualified for the Singapore 
Box 3 Singapore's legislative framework for quality

\section{Hospital/clinics}

- Private Hospitals and Medical Clinics Act (1980) - empowers $\mathrm{MOH}$ officials to inspect premises and ensure compliance with minimum standards

Professional registration and conduct

- Medical Registration Act (1997)

- Nurses and Midwives Act (1975)

- Dentists Act (1948)

- Pharmacists Registration Act (1985)

- empower the respective professional Councils and Boards to uphold professional standards and investigate complaints of professional misconduct.

- empower the professional Councils/Boards to remove those deemed unfit to practice from the Register.

Medical products, procedures and diseases

- Medical, Therapy, Education \& Research Act (1972)

- Termination of Pregnancy Act (1974)

- Medicines Act (1975)

- Infectious Disease Act (1976)

- Human Organ Transplant Act (1987)

- Radiation Protection Act (1992)

- spell out what the medical profession can and cannot do in relation to specific situations-for example, the authority to remove organs after death for transplantation under certain circumstances and the requirement for proper counselling prior to termination of pregnancy.

Medical Council's recognition award in CME. ${ }^{12}$ The government therefore decided to make CME compulsory in 2003, and proof of satisfactory CME participation is now a requirement for recertification. ${ }^{13}$ No doubt, attention will soon turn to nurses, dentists, and pharmacists as the respective professional boards have been asked by the $\mathrm{MOH}$ to introduce continuing professional education.

In 2002 the MOH created a new and expanded Health Regulation Division out of the former Medical Audit and Accreditation Unit, with broad responsibilities for licensing and accreditation, legislative enforcement, surveillance, clinical audit and quality assurance programme, including the implementation of clinical pathways and best practices in the management of diseases. At the same time, the government gave notice that "by the end of 2003 regulations under the Private Hospitals and Medical Clinics Act will be revised to stipulate the scope and depth of quality assurance and infection control activities required of hospitals. In addition, a system to detect and mitigate potential sources of medical errors would be put in place." ${ }^{\prime 11}$

The regulation of health products (such as medical devices and health supplements) comes under a new statutory board, the Health Sciences Authority (HSA), formed in 2001 from the amalgamation of five former national agencies (the Centre for Drug Evaluation, Institute of Science and Forensic Medicine, National Pharmaceutical Administration, Product Regulation Department and Singapore Blood Transfusion Service). Its function is to safeguard the quality, safety and efficacy of medicinal and health related products including traditional medicines, medical devices, and radiation devices.
In spite of its best efforts, however, two highly publicised cases of liver failure in 2002-one ending in death and the other necessitating a liver transplant-were linked to the consumption of imported slimming pills containing banned substances ${ }^{14}$ — soberly driving home the point that nothing is fool-proof.

\section{GOOD ORGANIZATION AND MANAGEMENT}

A major goal of Singapore's healthcare system is to provide effective and efficient care through its vertically and horizontally integrated healthcare institutions (see box 1). The latter are organised into two clusters of hospitals and specialist medical centres, each with its share of primary care government polyclinics. Each institution is headed by a CEO and each cluster has a group CEO who reports to a Board of Directors appointed by the MOH.

The stated aim of "clustering", introduced in 2000, was to foster healthy competition between the clusters and enable "seamless care" within each cluster. As an illustration of seamless care, patients from the primary care polyclinics can be admitted to the hospitals in the same cluster without having to be reassessed at the hospital. Similarly, polyclinic patients can now have quick, hassle free access to specialist care in the hospitals whenever requested by the referring doctor.

To forge greater linkages between private general practitioners (GPs) and the restructured hospital specialists, joint CME and "shared care" programmes have been implemented by both clusters. Under the programme for cancer care, for instance, the GPs provide screening for cancer, deliver public health education programmes, and co-manage the complications of cancer therapy (for example, palliative care). The institutions, on their part, facilitate easy and quick access to referrals and diagnostic investigations and concentrate on multidisciplinary team based inpatient care.

Private hospitals have also followed suit by incorporating team based care in partnership with referring GPs, and have introduced structured treatment protocols. They have also formed strategic alliances with centres of excellence overseas and adopted the "best practices" of these institutions.

\section{Box 4 Singapore's trajectory in quality and} patient safety

Significant changes in the last 5 years

- Participation in the Maryland Quality Indicator Project (QIP) (1999)

- Monitoring of patient satisfaction (2000)

- Adoption of disease management (2000)

- Implementation of evidence based clinical practice guidelines (2001)

- Generating awareness of medical errors (2002)

- Reporting of "near misses" (2002)

- Documentation of prevalence of medical errors (2003)

Changes that are still needed

- Strengthening of the evidence base for quality initiatives, e.g. through rigorous programme evaluation.

- Greater civil society involvement, e.g. voluntary accreditation.

- Greater patient empowerment, e.g. through greater transparency with respect to publishing of hospital quality indicators. 


\section{INTRODUCING MANDATORY CLINICAL INDICATORS}

Over the years there had been piecemeal monitoring by individual hospitals of a handful of clinical indicators such as unplanned readmissions and nosocomial infections, but a lack of standardization had prevented valid comparisons to be made between hospitals. In 2000 the $\mathrm{MOH}$ gave outcomes measurement a shot in the arm by mandating that all acute care public and private hospitals should participate in the Maryland Quality Indicator Project (QIP). ${ }^{15}$ Participation in the QIP involves monitoring a set of clinical quality indicators (see below) and benchmarking these indicators against national and international norms:

- Inpatient mortality.

- Perioperative mortality.

- Unscheduled return to operating theatre.

- Unscheduled readmission within 15 days.

- Unscheduled admission following ambulatory procedure.

- Inpatient admission following unscheduled returns to A\&E department.

- Device utilization and device associated infection in the ICU.

Participation in the QIP has allowed regular and comparative feedback to the hospitals in the form of quarterly reports and data analyses, enabling them to carry out more focused and targeted quality improvement actions. By keeping track of unscheduled returns/readmissions/admissions, for example, the hospitals are able to identify and monitor patients at high risk, leading to better implementation of discharge planning, coordination of community services, patient education and follow up plans for patients with particular illness at high risk of return/readmission/admission.

Accurate and timely information capture is, of course, a challenge and, in this regard, Singapore's extensive use of information technology in the healthcare sector is proving to be a great enabler. Since 1990 all health related organizations such as hospitals, polyclinics, insurance companies, pharmacies, and the Central Provident Fund Board (which administers the compulsory medical savings scheme Medisave and other government sponsored Medical Insurance schemes) have been linked by MediNet, an electronic data interchange system comprising five components-namely, central claims processing, national patient master index, procurement, information service, and notification. Electronic medical records (up and running in the bigger hospitals since 2000), together with case mix funding (introduced in 1999), are greatly facilitating the accurate capture of clinical data.

\section{MONITORING PATIENT SATISFACTION}

A first nationwide survey to gather feedback from patients recently discharged from public hospitals revealed some interesting results. Overall, $80 \%$ of patients were satisfied or very satisfied with their recent stay. It was found that outcome satisfaction and care satisfaction were consistently more important to patients than service satisfaction, in that order. However, overall satisfaction was most strongly determined by care satisfaction, followed by outcome satisfaction, with service satisfaction being the least important. Patients' experiences with doctors and nurses were the strongest predictors of care satisfaction. ${ }^{16}$

The wealth of information obtained by this study established a baseline for future comparisons. Although the detailed results have not been made public, the hospitals have found the comparative data to be useful for benchmarking, organization development, and strategic planning purposes, in addition to total quality management. Many have followed up with their own smaller scale patient satisfaction surveys targeted at improving service levels.

\section{INTRODUCING CLINICAL PRACTICE GUIDELINES AND DISEASE MANAGEMENT}

Two important initiatives have helped improve the continuity of care from primary prevention through to recovery, including long term follow up. Firstly, a series of evidence based clinical practice guidelines on various conditions commonly encountered in primary care practice have been disseminated to all medical practitioners in both the public and private sectors. These were put together by expert specialist panels under the auspice of the MOH. Secondly, a disease management approach has been adopted by all hospitals. This targets Singapore's major causes of mortality and morbidity-for example, cancer, heart disease, hypertension, stroke, diabetes, and asthma. ${ }^{17}$ Specially trained case managers also provide comprehensive advice on matters such as admission, discharge, and after care follow up including home help services for the disabled.

Such a condition focused, team based approach has led to a better understanding of the entire care process on the part of clinicians, administrators, and case managers. If nothing else, coming together to meet and discuss the care of individual cases and to re-engineer the patient care processes has been beneficial.

\section{MEDICAL ERRORS TO THE FORE}

Only within the last 3 years has the term "medical errors" gained currency in Singapore-thanks to media reporting of the landmark publication by the Institute of Medicine in 2000 of "To Err is Human" ${ }^{2}$ and the worldwide interest it generated. Again, the nudge has come from the $\mathrm{MOH}$ rather than the public or third party payers, and certainly not the medical profession. No one knows what the true incidence of medical errors is, but everyone knows it is certainly not zero.

In 2003 the $\mathrm{MOH}$ commissioned a comprehensive nationwide study that is ongoing and which will establish the incidence of iatrogenic injuries or adverse patient events in all hospitals. It is likely, however, that the results will not be made public. But if the statistics from America are to be believed and extrapolated to Singapore, as many as 1000 lives (or the equivalent of three jumbo jets full of passengers) would be lost through medical errors each year! ${ }^{18}$

The present practice is to convene an official Committee of Inquiry after a serious adverse event has come to light, but a new national system for non-punitive reporting of "near misses" is being developed. ${ }^{19}$ A number of seminars have been organised to generate awareness among medical practitioners. The Singapore Medical Association has responded with an internal task force to review the situation and to recommend steps to reduce the incidence of "medical accidents", patient complaints, and medical litigation. ${ }^{20}$

Fortunately, public trust in the medical profession is largely intact, despite the occasional high profile medical negligence lawsuit. The Singapore public is generally not as vociferous or litigious as their western counterparts. Most disputes are resolved by negotiations, arbitration, and out of court settlements. However, the situation may well change as the number of lawsuits has climbed from three in 1998 to seven in 1999 to 10 in $2000 .^{21}$

\section{DISCUSSION}

Singapore's experience demonstrates the importance of strong political commitment and institutional capacities for self-improvement as success factors in the implementation of a national quality health and patient safety programme. It also shows the inadequacy of relying on structural 


\section{Key messages}

- Quality of care in Singapore has moved from a narrow focus on structural approaches to a broader multidimensional concept involving the monitoring of clinical indicators and medical errors.

- Strong political commitment and institutional capacities for self-improvement have been important success factors for programme implementation.

- Benefiting from leading edge concepts emanating from the healthcare quality movements in America and Europe, Singapore's own experience could in turn hold lessons for other small and developing countries.

- What Singapore still lacks is a culture of rigorous programme evaluation, greater public involvement, and greater patient empowerment.

approaches or a narrow focus on service quality—for it was only when the multidimensional concept of quality of care ${ }^{22}$ was better understood that Singapore's national quality of care programme was able to move to a higher plane. Nevertheless, strong structures are important for it is doubtful that, without them to build upon, the more difficult tasks of measuring and managing clinical processes and outcomes could have been as smoothly and expeditiously implemented.

The fact that Singapore's quality initiatives have been strongly influenced by the leading edge concepts emanating from the healthcare quality movement in America and Europe reinforces the value of sharing and learning from international experiences. Singapore's own evolutionary journey and experiences could in turn hold lessons for other small and developing countries who may find Singapore's "intermediate" model easier to identify with than the more complex and pluralistic systems of the US and Europe. For instance, the menu of generic options as described in this article can easily be adapted to suit local conditions.

The lack of transparency with respect to publication of data on patient satisfaction and medical errors in Singapore could be considered a weakness, but this has more to do with the politicosocial context than with the intrinsic merits of the model. It is relevant to ask if openness is a precondition of a robust system or vice versa; but, regardless of one's position on this matter, it must be acknowledged that the lack of a strong research base and a culture of rigorous programme evaluation aimed at informing policy would constitute a weakness.

On the positive side, quality health care has undoubtedly come a long way in Singapore. Doctors are no longer practising medicine in a "black box", shielded from lay scrutiny. The processes, even if not completely transparent, are at least becoming translucent. Providers are audited by the government, which is itself accountable to the people.

The present phase should be seen as part of an ongoing change process. With further devolution of the $\mathrm{MOH}^{\prime} \mathrm{s}$ provider functions and eventually, perhaps, a clearer regulator-purchaser-provider split, the future could see more provider inspired initiatives and greater patient empowerment. A system of voluntary accreditation might even emerge as society grows and as Singaporeans rely less on a benevolent government wielding regulatory powers on their behalf to ensure the quality and safety of their health.

Finally, it must be acknowledged that there is only so much that external regulation can do to ensure ethical, safe, and quality health care. Quality, like integrity, must ultimately spring from within. The eventual goal is therefore for every care giver to internalize a value driven culture of quality care, safety consciousness, and public accountability-one in which quality becomes an incentive and not merely an end.

\section{REFERENCES}

1 Institute of Medicine. Crossing the quality chasm: a new health system for the 21 st century. Washington, DC: National Academy Press, 2001.

2 Kohn LT, Corrigan JM, Donaldson MS, et al. To err is human: building a safer health system. Washington, DC: National Academy Press, 2000.

3 Donabedian A. Evaluating the quality of medical care. Milbank $Q$ 1966:44:166-206.

4 Central Intelligence Agency. The World Fact Book 2001. Washington, DC: Central Intelligence Agency Office of Public Affairs, 2001.

5 Ministry of Health. Health Facts Singapore (available at: www.moh.gov.sg/ hfacts/hfacts. html).

6 Mouradian G. Quality revolution: a history of the quality movement. Lanham, MD: University Press of America, 2002.

7 Sashkin M, Kiser KJ. Putting total quality management to work. San Francisco: Berrett-Koehler, 1993.

8 Goldfield N, Pine M, Pine J. Measuring and managing health care quality: procedures, techniques, and protocols. New York: Aspen Publishers, 1996.

9 Donaldson MS, ed. Measuring the quality of health care. Washington, DC National Academy Press, 1999.

10 Chassin MR, Galvin RW and the National Roundtable on Health Care Quality. The urgent need to improve health care quality. JAMA 1998;280:1000-5.

11 Ministry of Health. Addendum to the President's Address. March 2002.

12 Chee YC. Address by the Master, Academy of Medicine, Singapore at the 1999 Annual Induction Dinner at the Regent Hotel. Ann Acad Med Singapore 1999;4:618-9.

13 Anon. Keep up or lose licence, doctors here told. The Straits Times. 20 July 2002:1.

14 Anon. Slim 10 victim Rani dies. The Straits Times Interactive . 2002 (available at http://straitstimes.asial.com.sg/breakingnews/story/ $0,1895,123372,00 . \mathrm{html}$.

15 Maryland QIP. Available at: www.hanys.org/tools/educate/qipbroch.htm.

$16 \mathrm{Ng} \mathrm{TP}$, Lim MK. Patient feedback survey. Applied Research Corporation, Singapore. 2000

17 Cheah J. Chronic disease management: a Singapore perspective. BMJ 2001;323:9990-3.

18 Tan SY. Blame the pilots, blame the doctors: lessons from SQ 006. Singapore Med J 2002;43:276

19 Ministry of Health Clinical Quality Branch. Making quality our way of life. Singapore Med Assoc News 2002;6:6-10.

20 Low CH. Presidents' Forum: Reducing medical accidents and improving patient complaints. Singapore Med Assoc Newsletter 2002;34(2).

21 Singapore High Court Registry 2002.

22 Palmer RH. Quality health care. JAMA 1996;275:1851-2.

23 Lim MK. Health care systems in transition. II. Singapore, Part I. An overview of health care systems in Singapore. J Public Health Med 1998;20:16-22.

24 World Health Organisation. Report 2000 health systems: improving performance. Geneva: World Health Organisation, 2000.

25 Anon. Singapore Tourist Board overseas visitors' survey, 2000.

26 Anon. Singapore faces new competitors in services. The Straits Times 24 January 2003:1. 